Article

\title{
All Titanium Microelectrode Array for Field Potential Measurements from Neurons and Cardiomyocytes-A Feasibility Study
}

Tomi Ryynänen ${ }^{1, *}$, Ville Kujala ${ }^{2}$, Laura Ylä-Outinen ${ }^{2}$, Ismo Korhonen ${ }^{2}$, Jarno M.A. Tanskanen ${ }^{3}$, Pasi Kauppinen ${ }^{3}$, Katriina Aalto-Setälä ${ }^{2,4}$, Jari Hyttinen ${ }^{3}$, Erja Kerkelä $^{2, \dagger}$, Susanna Narkilahti ${ }^{2}$ and Jukka Lekkala ${ }^{1}$

1 Department of Automation Science and Engineering, Tampere University of Technology, Korkeakoulunkatu 3, FI-33720 Tampere, Finland; E-Mail: jukka.lekkala@tut.fi (J.L.) Institute for Biomedical Technology, University of Tampere, Biokatu 12, FI-33520 Tampere, Finland; E-Mails: ville.kujala@uta.fi (V.K.); laura.yla-outinen@uta.fi (L.Y.-O.); ismo.korhonen@tut.fi (I.K.); katriina.aalto-setala@uta.fi (K.A.-S.); erja.kerkela@veripalvelu.fi (E.K.); susanna.narkilahti@uta.fi (S.N.)

3 Department of Biomedical Engineering, Tampere University of Technology, Biokatu 6, FI-33520 Tampere, Finland; E-Mails: jarno.m.tanskanen@tut.fi (J.M.A.T.); pasi.kauppinen@tut.fi (P.K.); jari.hyttinen@tut.fi (J.H.)

4 Heart Center, Tampere University Hospital, Biokatu 6, FI-33520 Tampere, Finland

$\dagger$ Current address: Finnish Red Cross Blood Service, Kivihaantie 7, FI-00310 Helsinki, Finland

* Author to whom correspondence should be addressed; E-Mail: tomi.ryynanen@tut.fi;

Tel.: +358-3-3115-11; Fax: +358-4219-198-1189.

Received: 19 September 2011; in revised form: 19 October 2011 / Accepted: 20 October 2011 / Published: 28 October 2011

\begin{abstract}
In this paper, we describe our all-titanium microelectrode array (tMEA) fabrication process and show that uncoated titanium microelectrodes are fully applicable to measuring field potentials (FPs) from neurons and cardiomyocytes. Many novel research questions require custom designed microelectrode configurations different from the few commercially available ones. As several different configurations may be needed especially in a prototyping phase, considerable time and cost savings in MEA fabrication can be achieved by omitting the additional low impedance microelectrode coating, usually made of titanium nitride (TiN) or platinum black, and have a simplified and easily processable
\end{abstract}


MEA structure instead. Noise, impedance, and atomic force microscopy (AFM) characterization were performed to our uncoated titanium microelectrodes and commercial TiN coated microelectrodes and were supplemented by FP measurements from neurons and cardiomyocytes on both platforms. Despite the increased noise levels compared to commercial MEAs our tMEAs produced good FP measurements from neurons and cardiomyocytes. Thus, tMEAs offer a cost effective platform to develop custom designed electrode configurations and more complex monitoring environments.

Keywords: microelectrode array (MEA); measurement noise; impedance; stem cell; field potential measurement; titanium

\section{Introduction}

In this paper, we introduce a relatively inexpensive method to fabricate custom microelectrode arrays (MEAs). Since the early 1970s, MEAs have been used [1], now for almost four decades, as powerful tools to measure field potentials (FPs) from various kinds of tissues and cell cultures, including neurons and cardiomyocytes. With MEAs it is possible to study the electrical activity on cell population level instead of single cells measured using patch clamp analysis.

MEA platforms have been widely used in neuroscience research. Acute tissue slices, primary cells as well as cell lines can be cultured on different types of MEAs and their neuronal electrical activities can be measured [2-5]. MEAs offer useful, non-invasive, repeatable, and long term setups for neuronal network activity measurements that can be used to study spontaneous activity, effects of electrical and chemical stimuli, and plasticity [6-9]. Additionally, they can be used for drug screening purposes and for toxicological studies [10,11]. Nevertheless, only a few MEA studies have been conducted with human-derived neurons. Especially for drug screening or tissue engineering purposes, the use of human-derived neurons $[6,12]$ would be important.

MEAs serve also as a valuable tool in studying the basic electrophysiology of cardiomyocytes [13]. MEAs have been used in conduction studies of cardiomyocytes derived from human embryonic stem cells (hESCs) [14], for analyzing cardiomyocytes derived from different hESC lines [15] and for studying in vitro pharmacology of the hESC-derived cardiomyocytes [15-18]. The field potential duration (FPD) on MEAs has been shown to correspond to the QT prolongation potential of different drugs indicating that the platform may be useful in safety assays [19].

Most of the current in vitro research can well be performed using the commercially available MEAs. Novel research questions including controlled growth of cell networks, complex and sensor controlled cell culturing environments for long term measurements, or just new material trials are, however, constantly increasing demand for custom designed MEAs. Especially the prototyping phase of new ideas would benefit from MEA platforms enabling fast and low cost customization.

When fabrication costs of a set of MEAs with several custom designed electrode configurations is considered, there are practically two ways to cut the costs. The first one is cutting the lithography mask costs, which can be achieved for example by reducing the area of the microlithographically patterned part [20,21], direct writing the custom patterns [22], or utilizing inexpensive film masks, which have 
been applied, even though not discussed, also in this paper. Another way to cut the fabrication costs and also time, is to keep the MEA structure as simple as possible. It is clear that substrate, metal layer including electrodes, contact pads, and tracks, and an insulator layer are needed. The necessity of one more common part in the MEA structure, the additional microelectrode coating, however, can be questioned.

Throughout MEA history, engineers have tried to improve the measurement and stimulation capabilities of the microelectrodes by applying an additional coating on a microelectrode base material. The coating is usually aimed to reduce noise and improve the impedance characteristics and charge transfer capacity of the microelectrodes, which affect the probability of detecting cellular activity and the stimulation capability. Wide availability and sufficiently good electrical characteristics have made platinum black (Pt black) probably the most commonly used microelectrode surface coating throughout MEA history $[1,4,23,24]$, even if it suffers from adhesion and reproducibility problems [25,26]. TiN is the microelectrode surface material favored by one of the leading commercial MEA manufacturers (Multi Channel Systems (MCS), Reutlingen, Germany) [27-29]. Iridium oxide (IrOx) is widely studied, even though not yet commercialized, microelectrode coating that has excellent charge transfer capacity and high long term stability, but suffers from need for electrochemical (re)activation $[25,26,30]$. There is no common opinion whether TiN or IrOx has better characteristics [26,28,31]. Recently several groups have reported microelectrodes coated with carbon nanotubes (CNTs) [32-35] which have not only superior electrical properties but also support cell adhesion via extremely rough surface [34]. There are, however, general concerns related to the biocompatibility [36] and mechanical stability [26,35] of the CNT coatings. Expensive materials and in some cases necessary special devices and special expertise for reactive or otherwise complex processes make the additional coating often the most expensive, time consuming, and error prone phase in the MEA fabrication — an issue which can be ignored at most by very experienced and well equipped organizations. Thus if the sufficient MEA performance level can be reached without the additional coating, it may lead to significant cost and time savings in MEA fabrication.

MEAs with gold, platinum, or indium tin oxide (ITO) microelectrodes without additional coating are commercially available [20], but certain aspects, e.g., material costs and processing difficulties, make those materials less attractive for simple and low cost fabrication schemes. Titanium, on the other hand, is a common, easy to process, and highly biocompatible electrode material. Titanium has not, however, been employed as sole MEA microelectrode material, but always either only as thin adhesion layer for some other metal [2,3,24,30] or coated with titanium nitride (TiN) [27]. The rationale against using titanium microelectrodes without additional coating may be the existence of a few nanometers thick dielectric native oxide $\left(\mathrm{TiO}_{2}\right)$ layer which always forms on a titanium surface in air. However, direct tunneling through the thin dielectric layer [37], existence of conducting suboxides [37-39], and impurities originating from the glass surface prior to titanium deposition [38] have been proposed to account for local electron transfer through the native oxide layer, thus giving rise to sufficient conductivity.

The aim of this study is to show that the FP measurement capabilities of titanium microelectrodes without any additional surface coating are well comparable to standard microelectrodes used today. As shown in this paper, with our in-house MEAs (later referred to as tMEAs), such microelectrodes are 
fully capable for FP measurements from neurons and cardiomyocytes, yielding signal quality well sufficient for the intended analysis purposes.

\section{Methods}

\section{1. tMEA Fabrication}

Our in-house built tMEAs have 58 microelectrodes in $8 \times 8$ square matrix format, with one microelectrode missing from each corner and two microelectrodes missing from both sides close to the central line. There are two large electrodes on both sides of the matrix which can be used as measurement reference, ground, and stimulation electrodes. In the first batch of tMEAs (later referred to as old tMEAs, because of being used in biological measurements before noise and impedance measurements), all the microelectrodes were square shaped and approximately $30 \times 30 \mu \mathrm{m}$ in size. The second batch of tMEAs (later referred to as new tMEAs, because not used in biological measurements before other measurements) suffered from certain processing condition failures causing microelectrode size variation and rounding of the intended square shape with the average microelectrode diameter of $23 \mu \mathrm{m}$. To verify noise performance vs. electrode area dependence, some of the microelectrodes were made larger ( $55 \mu \mathrm{m}$ in diameter). The inter-electrode distance was $200 \mu \mathrm{m}$ in all tMEAs.

The fabrication process of tMEAs is illustrated in Figure 1. Briefly, a $0.9 \mathrm{~mm}$ thick Schott Desag D263 glass (Schott, Mainz, Germany) was chosen for the substrate material due to its high mechanical durability. The glass was cut into $49 \times 49 \mathrm{~mm}$ size wafers, cleaned with dishwashing liquid, water and ethanol, and e-beam coated with $300 \mathrm{~nm}$ layer of titanium at Oplatek Oy (Leppävirta, Finland). Before proceeding to the next lithographic steps, the glass wafers were cleaned with acetone, 2-propanol, and de-ionized water. Spin coated hexamethyldisilazane (HMDS, Acros Organics, Geel, Belgium) was used as photoresist adhesion promoter followed with immediate spin coating of ma-P 1225 positive photoresist (micro resist technology, Berlin, Germany) to $\sim 2.2 \mu \mathrm{m}$ thickness that was further baked on the wafers. Next, the microelectrode pattern from laser photoplotted mask was UV-exposed, developed, and hardbaked on photoresist.

Figure 1. Fabrication process of tMEAs: (a) Bulk glass wafer; (b) Titanium coating; (c) Photoresist coating; (d) UV-exposure and development; (e) Wet etching and resist removal; (f) PECVD deposition of $\mathrm{Si}_{3} \mathrm{~N}_{4}$; (g) Photoresist coating; (h) UV-exposure and development; and (i) Dry etching and resist removal. Images are not to scale.

a)

b)

c)

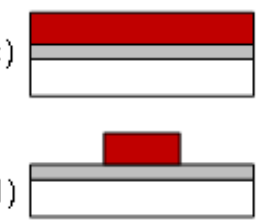

e)

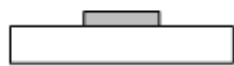

f)

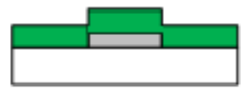

g)

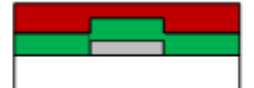

h)

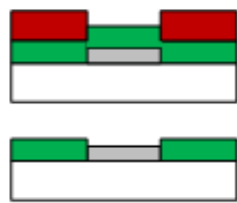

i)

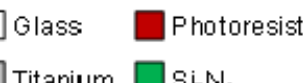

$\square$ Titanium $\square S_{3} N_{\mathbf{l}}$ 
Wet etching $\left(20 \mathrm{H}_{2} \mathrm{O}: 1 \mathrm{H}_{2} \mathrm{O}_{2}(30 \%)\right.$ : $\left.1 \mathrm{HF}\right)$ was used to transfer the microelectrode pattern from the photoresist layer to titanium. After cleaning the wafer from remaining photoresist, a $500 \mathrm{~nm}$ layer of silicon nitride $\left(\mathrm{Si}_{3} \mathrm{~N}_{4}\right)$ was PECVD deposited as dielectric insulating layer at Optoelectronics Research Centre (ORC, Tampere University of Technology, Tampere, Finland). Lithographic steps for making openings for electrodes and contact pads in the insulator layer were performed with the same procedures as the patterning of titanium described above. After the hardbake step, however, reactive ion etching with $\mathrm{SF}_{6}$ and $\mathrm{O}_{2}$ gases [40] was applied instead of wet etching. In final fabrication step, a short oxygen plasma treatment $\left(\mathrm{O}_{2} 30 \mathrm{sccm}\right.$, RF power $30 \mathrm{~W}$, pressure $\left.30 \mathrm{mTorr}\right)$, was run in reactive ion etcher (RIE, Advanced Vacuum Vision 320, Advanced Vacuum, Lomma, Sweden) to make the surface hydrophilic.

\subsection{Commercial MEAs Used for Comparison}

The commercial MEAs (later referred to as cMEAs) used in the study were standard MEAs of type 200/30iR-Ti (MCS). The 59 round microelectrodes in $8 \times 8$ square format had the diameter of $30 \mu \mathrm{m}$ and the inter-electrode distance of $200 \mu \mathrm{m}$. Titanium was used as a conductor base material and, unlike in tMEAs, both the electrodes and the contact pads were coated with TiN. Alike in tMEAs, $500 \mathrm{~nm}$ PECVD silicon nitride was used as an insulator layer [27]

\subsection{Noise Measurements}

Noise and biological FP measurements were performed with MEA1060-Inv-BC amplifier and MC_Rack software, both from MCS. The noise signal was recorded from MEAs filled with cardiomyocyte cell culture medium (EB-medium) without cells, at first for three minutes immediately after filling the MEAs, and after one and two hours to see how the noise behaves as a function of time. The measurement sampling frequency was $20 \mathrm{kHz}$. Total of three new and three old tMEAs and three new and three old cMEAs were measured.

From each MEA, 6 microelectrodes from the second row were chosen for noise analysis. From the data, we calculated root mean square (RMS) noise for each microelectrode by using the following formula:

$$
R M S=\sqrt{\frac{1}{n} \sum_{i=1}^{n} x_{i}^{2}}
$$

where $n$ is the number of samples in the measured signal and $x_{i}$ is the voltage sample measured at time $i$. For each MEA, an average RMS noise was calculated. Thereafter, average RMS noise for each MEA type was calculated from the average RMS noises of individual MEAs. As it has been shown that the noise of microelectrodes is inversely related to the square root of the electrode area [41], for fair comparisons between tMEAs and cMEAs, the RMS noise levels of tMEA microelectrodes were normalized to correspond to the RMS noise level of a microelectrode with the area equal to the area of a cMEA microelectrode.

Noise voltage spectra over the frequency range from $0.3 \mathrm{~Hz}$ to $10 \mathrm{kHz}$ were plotted using the Welch estimate method [42] with the 50\% overlapping and window length of 60615 samples. Estimates were calculated with a Measurement Signal Processing (MSP) toolbox [43] for Matlab. Also noise voltage histograms with 200 bins of width $20 \mu \mathrm{V}$ were calculated for the microelectrodes. 


\subsection{Impedance Measurements}

Impedances of the tMEA and cMEA microelectrodes were measured using Solartron Analytical 1260A Impedance/Gain-phase Analyzer (Solartron Analytical, Hampshire, UK) connected to Solartron 1294A Impedance Interface, whose four-terminal non-human interface was utilized, and connected according to the two-terminal impedance measurement configuration described in [44]. 1294A was connected to a MEA via MEA1060-Inv contacting adapter (MCS). The MEAs were let stabilize at least over night at room temperature in isotonic saline (sodium chloride $9 \mathrm{mg} / \mathrm{mL}$, Baxter, Lessines, Belgium), rinsed with distilled water, and let stabilize for at least one hour filled with the cardiac cell culture medium (described in detail in Section 2.6) prior to impedance measurement. The contact pins of the contacting adapter and the MEA contact pads were cleaned with $70 \%$ ethanol. The temperature of a MEA was allowed to stabilize to approximately $37^{\circ} \mathrm{C}$ for at least three minutes in the contacting adapter. Impedance measurement current was set to $10 \mu \mathrm{A}$. Current driven measurement setup was selected to ensure that the current density remained sufficiently low (approx. $14 \mathrm{~mA} / \mathrm{mm}^{2}$ for a microelectrode diameter of $30 \mu \mathrm{m}$ ) regardless of microelectrode impedances.

Impedances were measured at $1 \mathrm{kHz}$, which is the common practice also in commercial MEA datasheets, and data was recorded using SMaRT software (Solartron Analytical). With tMEAs, the ground electrode on the same side of the MEA as in the cMEAs was used. For fair comparison between tMEAs and cMEAs, the impedance magnitudes of the tMEA microelectrodes were normalized by the microelectrode surface areas so that all impedance magnitudes are presented for microelectrodes with the surface area of cMEA microelectrodes. In the sequel, this is referred to as area-normalization.

As one potential source for lower impedance is the bigger effective surface area, we also performed atomic force microscopy (AFM) measurements (XE-100 AFM, Park Systems, Suwon, Korea) to assess effective surface area difference between titanium and TiN surfaces. For each MEA type $1 \mu \mathrm{m} \times 1 \mu \mathrm{m}$ scans including $256 \times 256$ pixels were performed in a tapping mode.

\subsection{Assessment of Neuronal Cell FP Measurement Capabilities}

The neural differentiation of hESCs was performed as reported $[45,46]$ and MEA preparation and measurements were performed as described earlier [6]. Prior to cell seeding, the MEAs were coated with two step coating procedure with $0.05 \%(\mathrm{w} / \mathrm{v})$ polyethylenimine (PEI) and mouse laminin, $20 \mu \mathrm{g} / \mathrm{mL}$ (both from Sigma-Aldrich, St. Louis, MO, USA). Thereafter, neurospheres, predifferentiated from hESCs for 8 weeks, were cut into small aggregates $(\varnothing \sim 100 \mu \mathrm{m})$ and seeded onto coated MEA plates. Neuronal culture consisted of 1:1 DMEM/F12 and Neurobasal media supplemented with $2 \mathrm{mM}$ GlutaMax, $1 \times \mathrm{B} 27,1 \times \mathrm{N} 2$ (all from Invitrogen, Carlsbad, CA, USA), and $25 \mathrm{U} / \mathrm{mL}$ penicillin/streptomycin (Lonza, Basel, Switzerland). The medium was changed 3 times per week. The cells were cultured for 21 days in vitro (DIV) on three tMEAs and on three cMEAs. Spontaneous activities of neuronal networks were measured for five minutes once a week. Neuronal action potential spike detection was performed based on an amplitude threshold at five times the signal standard deviation from the mean of the signal. Also, phase contrast microscope images were taken weekly. 


\subsection{Assessment of Cardiomyocyte FP Measurement Capabilities}

Differentiation of hESC line $\mathrm{H} 7$ was performed as described earlier [47]. The MEAs were coated as follows: 30 minutes with fetal bovine serum (Gibco, Invitrogen) after which they were washed twice with sterile water and one hour with $0.1 \%$ gelatine type A (Sigma-Aldrich). After coating the MEAs, the spontaneously beating cardiomyocyte aggregates, excised from cell cultures mechanically, were plated onto the electrode areas in cell culture medium consisting of KO-DMEM (Invitrogen) supplemented with 20\% FBS (Invitrogen), 1\% NEAA (Lonza), 1\% Glutamax (Invitrogen), and 50 U/mL penicillin/streptomycin (Lonza). The cells were cultured for 14 days in vitro on four tMEAs and on three cMEAs. After 14 days in culture on MEA, baseline electrical signal was recorded and the effect of E-4031 (Alomone Labs, Jerusalem, Israel), a known blocker of human ether-a-go-go potassium current at the concentration of $300 \mathrm{nM}$ was analyzed in order to test whether tMEAs were sensitive enough to detect changes in the cardiac repolarization current.

\section{Results and Discussion}

\section{1. tMEA Fabrication}

By omitting additional microelectrode coating we were able to save at least one deposition step compared to Pt black coated microelectrodes [24] and one deposition and one lift-off process step, compared to TiN coating [29]. Depending on the selected fabrication method, CNT and IrOx coatings might need even more process steps. Cutting process steps does not only save time and money, but decreases the risk of process failures and increases the repeatability of the fabrication process. In the case of integrating sensors or cell growth guiding structures on MEAs or replacing chemically and thermally durable glass substrate or silicon nitride insulator layer by some polymer based materials $[48,49]$, each additional process step might be even fatal for the most sensitive layers or force to make unpractical changes in the fabrication process chain.

\subsection{Noise Analysis}

Even if precise statistical analysis was omitted due to difficulty of defining the exact area of each individual arbitrary shaped microelectrode, a rough comparison of RMS noise levels between the normal approximately $23 \mu \mathrm{m}$ microelectrodes and $55 \mu \mathrm{m}$ control microelectrodes of new tMEAs allowed us to conclude that at least on average, the tMEAs follow the theory of the noise of electrodes being inversely related to the square root of the electrode area [41]. Thus, we were able to normalize the RMS noise data of tMEAs to correspond the electrode size of cMEAs, i.e., $30 \mu \mathrm{m}$ in diameter.

The average measured RMS noises both from three new and three old tMEAs and cMEAs with average deviations along with the area normalized average RMS noises of tMEAs are all shown in Table 1 . The results indicate roughly 33\% higher noise for the new tMEAs compared to new cMEAs and roughly $90 \%$ higher noise for the old tMEAs compared to old cMEAs. The lower noise of old MEAs compared to new MEAs is probably mainly due to electrode surface modifications caused by long term exposure to cell and culture medium. At least for tMEAs, but possibly also for cMEAs, the batch to batch variations partly explain the noise variations. The uncontrolled long term natural 
oxidation of titanium may partly explain the ten times larger deviation in average RMS noise from MEA to MEA observed in tMEAs compared to cMEAs. Another explanation for the larger deviation is tMEAs' presumably bigger deviation also on microelectrode sizes and other MEA structure dimensions compared to cMEAs.

Table 1. Average RMS noise and impedance magnitudes (area-normalized) comparison of three new (unused) and three old (previously used in biological measurements) tMEAs and cMEAs at three different times after filling the MEAs with medium.

\begin{tabular}{|c|c|c|c|c|c|c|c|c|c|}
\hline $\begin{array}{c}\text { Time } \\
\text { (h) }\end{array}$ & $\begin{array}{c}\text { Average } \\
\text { measured } \\
\text { RMS } \\
\text { noise } \\
(\mu \mathrm{V})\end{array}$ & $\begin{array}{c}\text { Average } \\
\text { noise } \\
\text { deviation } \\
(\mu \mathrm{V})\end{array}$ & $\begin{array}{c}\text { Average } \\
\text { normalized } \\
\text { RMS noise } \\
(\mu \mathrm{V})\end{array}$ & $\begin{array}{c}\text { Average } \\
\text { normalized } \\
\text { impedance } \\
\text { magnitude } \\
\text { at } 1 \mathrm{kHz} \\
(\mathrm{k} \Omega)\end{array}$ & $\begin{array}{c}\text { Average } \\
\text { normalized } \\
\text { impedance } \\
\text { magnitude } \\
\text { deviation } \\
(\mathrm{k} \Omega) \\
\end{array}$ & $\begin{array}{c}\text { Average } \\
\text { measured } \\
\text { RMS } \\
\text { noise } \\
(\mu \mathrm{V})\end{array}$ & $\begin{array}{c}\text { Average } \\
\text { noise } \\
\text { deviation } \\
(\mu V)\end{array}$ & $\begin{array}{c}\text { Average } \\
\text { normalized } \\
\text { impedance } \\
\text { magnitude } \\
\text { at } 1 \mathrm{kHz} \\
(\mathrm{k} \Omega)\end{array}$ & $\begin{array}{c}\text { Average } \\
\text { normalized } \\
\text { impedance } \\
\text { magnitude } \\
\text { deviation } \\
(\mathrm{k} \Omega)\end{array}$ \\
\hline & \multicolumn{5}{|c|}{ New tMEA } & \multicolumn{4}{|c|}{ New cMEA } \\
\hline 0 & 8.3 & 3.7 & 7.5 & - & - & 5.7 & 0.3 & - & - \\
\hline 1 & 7.1 & 1.1 & 6.4 & 126.6 & 39.1 & 4.9 & 0.3 & 54.8 & 1.0 \\
\hline \multirow[t]{2}{*}{2} & 7.1 & 2.1 & 6.3 & - & - & 4.7 & 0.3 & - & - \\
\hline & \multicolumn{5}{|c|}{ Old tMEA } & \multicolumn{4}{|c|}{ Old cMEA } \\
\hline 0 & 5.1 & 1.6 & 5.8 & - & - & 2.7 & 0.2 & - & - \\
\hline 1 & 4.3 & 2.7 & 4.8 & 68.0 & 20.4 & 2.7 & 0.1 & 42.5 & 2.5 \\
\hline 2 & 4.3 & 1.7 & 4.8 & - & - & 2.7 & 0.1 & - & - \\
\hline
\end{tabular}

Figure 2. (a) Typical noise voltage density spectra of old tMEA and cMEA microelectrodes at times of $0 \mathrm{~h}, 1 \mathrm{~h}$, and $2 \mathrm{~h}$ after filling the MEAs with medium. Noise voltage histograms (200 bins of width $0.2 \mu \mathrm{V}$ ) at the time of $1 \mathrm{~h}$ time point are presented from the same (b) tMEA and (c) cMEA microelectrodes.

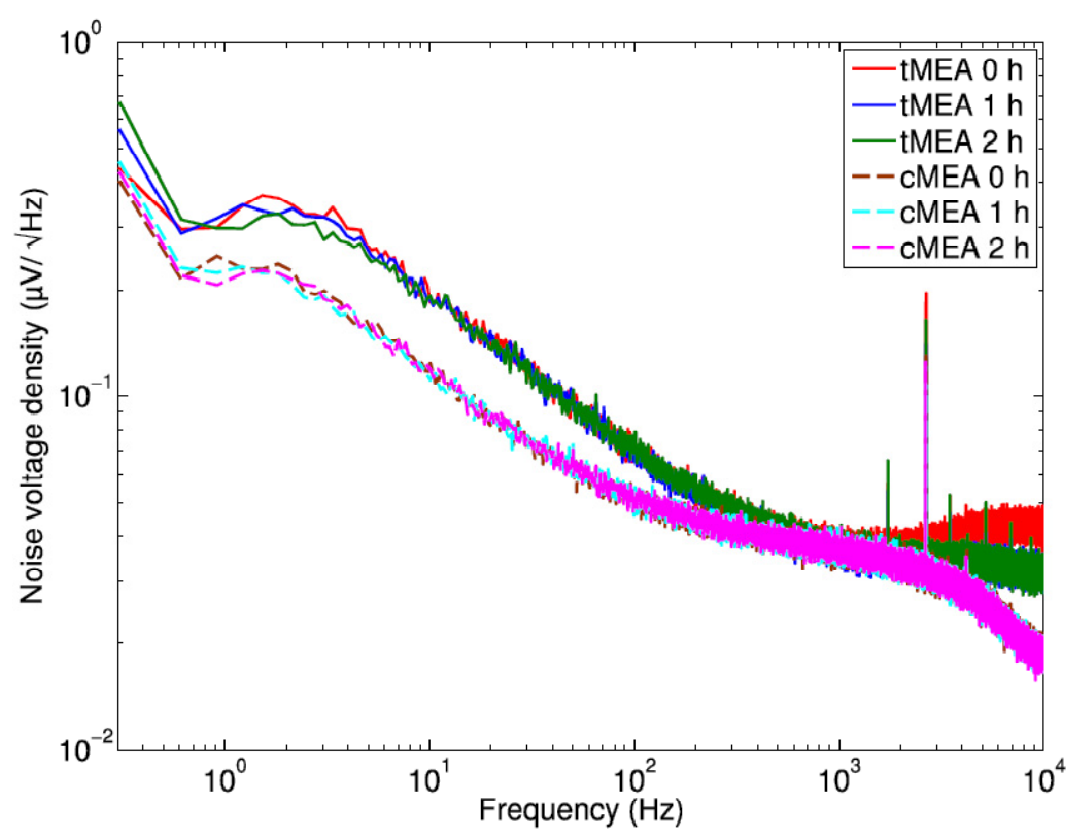

(a) 
Figure 2. Cont.

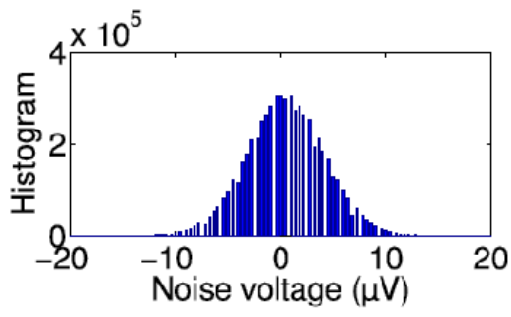

(b)

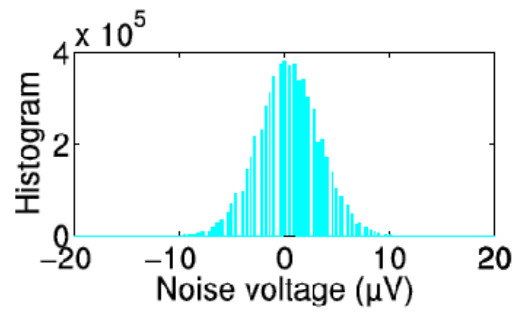

(c)

Typical noise voltage spectra are shown in [Figure 2(a)] from one microelectrode of an old tMEA and an old cMEA 0,1 , and $2 \mathrm{~h}$ after filling the MEAs with medium. [Figure 2(b,c)] include 200 bin noise voltage histograms from the same microelectrodes at the $1 \mathrm{~h}$ time point. Both MEA types have quite constant spectrum behavior as a function of time over the full frequency range excluding the clear drop at very low frequency between $0 \mathrm{~h}$ and $1 \mathrm{~h}$ in tMEA's spectrum. At about 2,640 Hz, both MEA types exhibit a peak, which we assume to originate from the amplifier electronics. In general some microelectrodes of some MEAs of all types also exhibited other occasional peaks, induced by some random additional activity in the laboratory or some MEA or microelectrode specific defects. Observable of $50 \mathrm{~Hz}$ mains frequency peak was more common in tMEAs than cMEAs. The shape of the histograms resembles in both cases Gaussian, but as can be assumed, the distribution for the tMEAs is wider.

\subsection{Impedance Magnitude Analysis}

Average impedances measured at $1 \mathrm{kHz}$ and normalized by the microelectrode area relative to that of the cMEA electrodes are given in Table 1 in conjunction with the corresponding average RMS noise values. The impedances of the tMEAs are on the average $60 \%$ higher for old MEAs and 130\% higher for new MEAs than those of the corresponding cMEAs.

The impedance magnitude values measured for cMEAs are at the same level with the earlier reported values [27-29]. As no previous reports about titanium MEAs exist, there is no direct comparison to earlier results corresponding to tMEAs. However one order of magnitude difference in impedance has been reported for uncoated and TiN coated gold microelectrodes [28,29], and Pt and ITO microelectrodes of the corresponding size have been reported having impedances above $800 \mathrm{k} \Omega$ [20]. Even if different studies are not fully comparable due to obvious differences in impedance measurement arrangements and microelectrode sizes, still the impedance characteristics of titanium microelectrodes can be considered competitive to other single material microelectrodes. Typical AFM images of the microelectrode surface of tMEA and cMEA are presented in Figure 3. Due to the columnar morphology of the TiN coating $[27,28]$ the AFM cantilever is able to measure only the very top surface of the TiN coating, giving no more than $10 \%$ increase in the effective surface area compared to the uncoated titanium. Thus, we suspect that our AFM has failed to convey full information of the fine surface structures, resulting in underestimating the effective surface area difference between tMEAs and cMEAs. The differences in noise and impedance values between tMEAs and cMEAs result from differences in effective surface areas due to different surface microstructures, and from different surface electrochemistries, including the natural oxidation of titanium. 
Figure 3. AFM images from (a) all-titanium microelectrode of tMEA and (b) TiN coated microelectrode surface of cMEA. Note the different vertical z-axis scales.
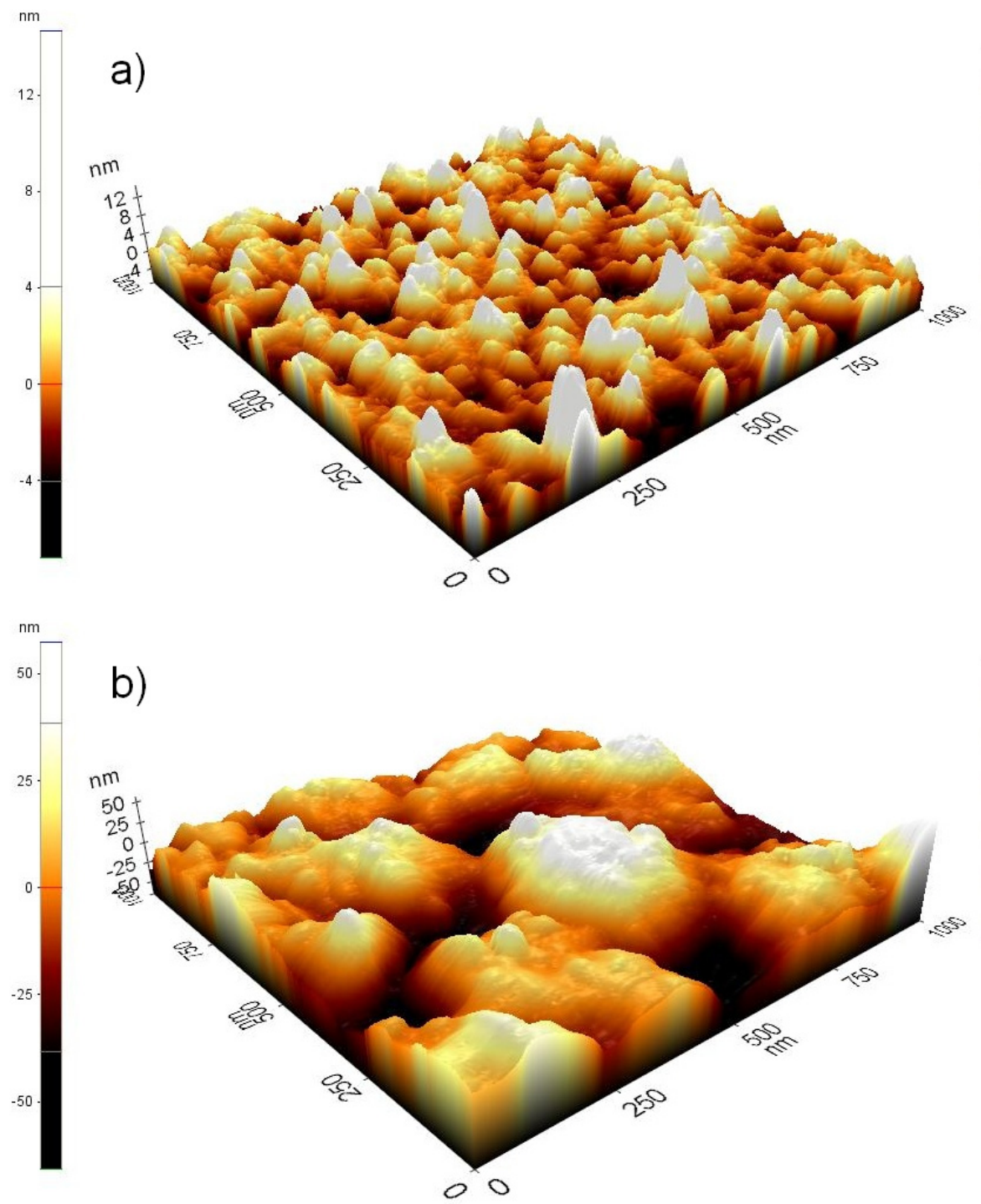

\subsection{Viability and Electrical Signaling of Neurons on tMEAs}

The neuronal aggregates attached successfully to all MEAs [Figure 4(a,c)]. The viability of the cells was similar on both tMEAs and cMEAs. Neurons started to grow processes and some cells migrated along these processes. In more detail, neuronal cells formed neural networks on tMEA surface and the spontaneous activity of the networks was measured with titanium microelectrodes [Figure 4(b)]. Corresponding spontaneous activity observed with cMEAs is presented in [Figure 4(d)]. Similarly, during the first week of culturing on MEA, the first individual spikes were detected on both MEA types. Further, the signaling developed as shown earlier [6] into more organized train-like activity [Figure 4(b,d)] and even burst activity. tMEAs had twice as high noise levels as the cMEAs, but the spikes were twice as high too. Thus tMEAs showed signal-to-noise (S/N) ratio comparable to cMEAs. The results support all-titanium MEAs' capability to function as an efficient and tunable tool for measuring neuronal activity and maturation with different electrode layouts. 
Figure 4. Neuronal and cardiac electrical activities on old MEAs. Neuronal network formed (a) on tMEA and (c) on cMEA. Train-like activity (b) from tMEA and (d) from cMEA. Signals were measured after 2 weeks culturing on MEAs. Note the different $y$-axis scale in figures (b) and (d); (e) Cardiac FP activity on tMEA over the two-week culture period. Note the increasing FP amplitudes; (f) 50\% prolonged FPD by E-4031 hERG channel blocker, detected with tMEA. The arrows point out the ends of the repolarizations.

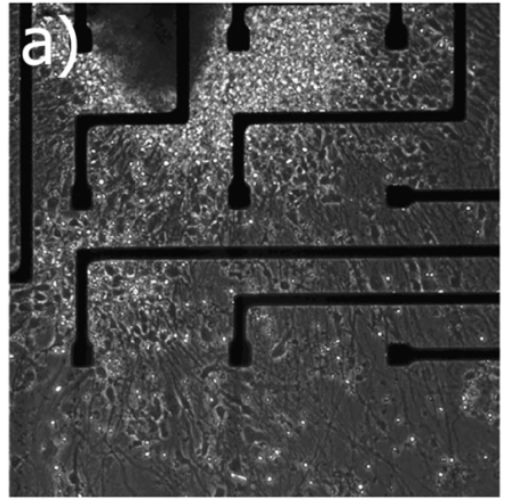

\section{b)}
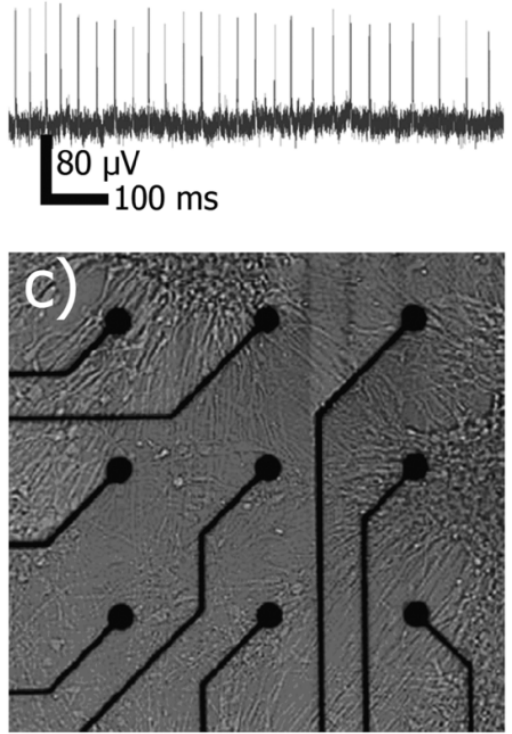

d)

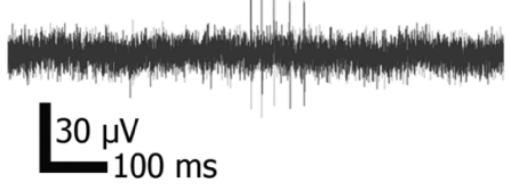

e)
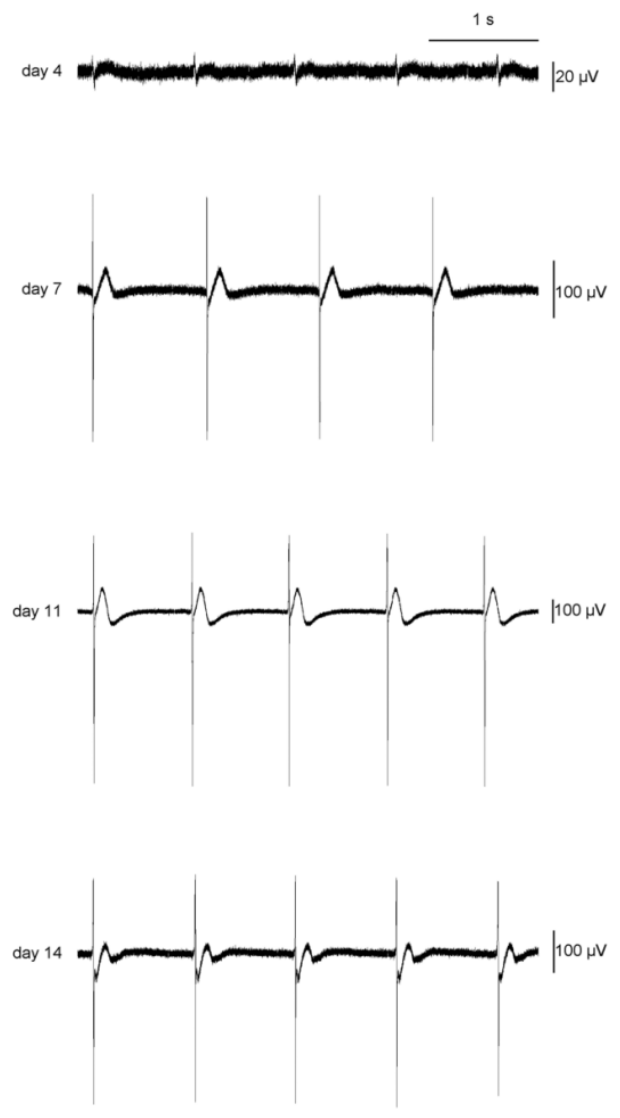

f)

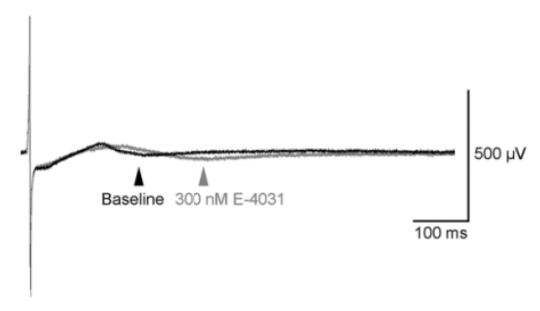

\subsection{Viability and Electrical Signaling of Cardiomyocytes on tMEAs}

The beating aggregates adhered well on tMEAs and no difference in adhesion property was detected compared to aggregates seeded on cMEAs. Upon inspection, the cardiomyocyte aggregates exhibited strong spontaneous contractility. We were able to record cardiac FP activity using tMEAs [Figure 4(e)]. The highest quality signals recorded were in the millivolt range. The FP signal generated by the spontaneously beating hESC-derived cardiomyocytes, was detected well with both tMEAs and cMEAs 
from DIV 4 onward, which was the first day of recording. The signal amplitude grew larger over time reaching the peak value at DIV 7. The morphology of the signal was also better defined by that time, marked by a higher $\mathrm{S} / \mathrm{N}$ ratio, which was a direct result of the larger systolic peak amplitude. This indicated stronger cell adhesion of the cardiomyocytes to the MEAs and better and more mature connection between cells. tMEAs tended to have greater variation in background noise levels between recordings of the same MEA than cMEAs. Also, a cardiac drug effect could be adequately and repeatedly recorded with tMEA. The hERG channel blocker E-4031, known to prolong QT time and FPD, prolonged FPD approximately 50\% [Figure 4(f)]. All this indicates as a proof-of-concept that all-titanium MEAs can be used for drug testing and that the resolution of the signal generated is good enough to detect changes in FP morphology.

\section{Conclusions}

Our experiments with hESC-derived neurons and cardiomyocytes show that the performance of all-titanium MEA microelectrodes is comparable to MEAs with TiN coated microelectrodes when the recording of FP signals from neuronal cells or cardiomyocytes is considered. For certain type of applications requiring detection of very weak signals or exact signal morphology, all-titanium MEA, despite its comparable $\mathrm{S} / \mathrm{N}$ ratio, may not be the optimal choice because of higher background noise and fluctuations. Furthermore, as the impedance measurement indicates, also the charge transfer capacity of titanium electrodes may have to be improved by some additional electrode surface coating for stimulation purposes, especially for cardiac cells - even though this was not fully evaluated in this paper. However, for many applications such as neuronal or cardiac activity, maturation, or drug testing where small additional noise is not an issue, the performance of all-titanium MEAs is adequate to detect neuronal spiking or changes in the cardiomyocyte FP morphology. The all-titanium technology presents us with several advantages in terms of modifying the MEA platform for future uses. These include being able to design and manufacture a variety of MEA layouts and microelectrode configurations in a cost and time effective manner e.g., for more complex experimental setups of neuronal network geometries or cardiomyocyte syncytium and connectivity studies.

\section{Acknowledgements}

This work was supported by the Academy of Finland (decision numbers 122947, 122959, 123233, 123359 and 123762), the Competitive Research Funding of the Tampere University Hospital, the Finnish Cultural Foundation, CHEMSEM graduate school, BioneXt Tampere, and Biosensing Competence Centre, BCC. The authors wish to thank all the current and past Stemfunc project members for the valuable comments and aid during the process towards this publication.

\section{References}

1. Thomas, C.A.; Springer, P.A.; Loeb, G.E.; Berwald-Netter, Y.; Okun, L.M. A miniature microelectrode array to monitor the bioelectric activity of cultured cells. Exp. Cell. Res. 1972, 74, 61-66. 
2. Gross, G.W.; Reiske, E.; Kreutzberg, G.W.; Mayer, A. A new fixed-array multimicroelectrode system designed for long-term recording of extracellular single unit activity in vitro. Neurosci. Lett. 1977, 6, 101-105.

3. Gross, G.W. Simultaneous single unit recording in vitro with a photoetched laser deinsulated gold multi-microelectrode surface. IEEE Trans. Biomed. Eng. 1979, 26, 273-279.

4. Pine, J. Recording action potentials from cultured neurons with extracellular microcircuit electrodes. J. Neurosci. Methods 1980, 2, 19-31.

5. Johnstone, A.F.M.; Gross, G.W.; Weiss, D.G.; Schroeder, O.H.-U.; Gramowski, A.; Shafer, T.J. Microelectrode arrays: A physiologically based neurotoxicity testing platform for the $21 \mathrm{st}$ century. Neurotoxicology 2010, 31, 331-350.

6. Heikkilä, T.J.; Ylä-Outinen, L.; Tanskanen, J.M.; Lappalainen, R.S.; Skottman, H.; Suuronen, R.; Mikkonen, J.E.; Hyttinen, J.A.; Narkilahti, S. Human embryonic stem cell-derived neuronal cells form spontaneously active neuronal networks in vitro. Exp. Neurol. 2009, 218, 109-116.

7. Illes, S.; Fleischer, W.; Siebler, M.; Hartung, H.P.; Dihne, M. Development and pharmacological modulation of embryonic stem cell-derived neuronal network activity. Exp. Neurol. 2007, 207, 171-176.

8. Jimbo, Y.; Tateno, T.; Robinson, H.P. Simultaneous induction of pathway-specific potentiation and depression in networks of cortical neurons. Biophys. J. 1999, 76, 670-678.

9. Wagenaar, D.A.; Pine, J.; Potter, S.M. An extremely rich repertoire of bursting patterns during the development of cortical cultures. BMC Neurosci. 2006, 7, 11.

10. Bal-Price, A.K.; Hogberg, H.T.; Buzanska, L.; Lenas, P.; van Vliet, E.; Hartung, T. In vitro developmental neurotoxicity (DNT) testing: Relevant models and endpoints. Neurotoxicology 2010, 31, 545-554.

11. Ylä-Outinen, L.; Heikkilä, T.; Skottman, H.; Suuronen, R.; Äänismaa, R.; Narkilahti, S. Human cell-based micro electrode array platform for studying neurotoxicity. Front. Neuroeng. 2010, 3, 111.

12. Buzanska, L.; Zychowicz, M.; Ruiz, A.; Ceriotti, L.; Coecke, S.; Rauscher, H.; Sobanski, T.; Whelan, M.; Domanska-Janik, K.; Colpo, P.; et al. Neural stem cells from human cord blood on bioengineered surfaces - Novel approach to multiparameter bio-tests. Toxicology 2010, 270, 35-42.

13. Reppel, M.; Pillekamp, F.; Lu, Z.J.; Halbach, M.; Brockmeier, K.; Fleischmann, B.K.; Hescheler, J. Microelectrode arrays: A new tool to measure embryonic heart activity. J. Electrocardiol. 2004, 37, 104-109.

14. Kehat, I.; Gepstein, A.; Spira, A.; Itskovitz-Eldor, J.; Gepstein, L. High-resolution electrophysiological assessment of human embryonic stem cell-derived cardiomyocytes: A novel in vitro model for the study of conduction. Circ. Res. 2002, 91, 659-661.

15. Pekkanen-Mattila, M.; Kerkelä, E.; Tanskanen, J.M.A.; Pietilä, M.; Pelto-Huikko, M.; Hyttinen, J.; Skottman, H.; Suuronen, R.; Aalto-Setälä, K. Substantial variation in the cardiac differentiation of human embryonic stem cell lines derived and propagated under the same conditions-A comparison of multiple cell lines. Ann. Med. 2009, 41, 360-370.

16. Braam, S.R.; Tertoolen, L.; van de Stolpe, A.; Meyer, T.; Passier, R.; Mummery, C.L. Prediction of drug-induced cardiotoxicity using human embryonic stem cell-derived cardiomyocytes. Stem Cell Res. 2010, 4, 107-116. 
17. Caspi, O.; Itzhaki, I.; Arbel, G.; Kehat, I.; Gepstien, A.; Huber, I.; Satin, J.; Gepstein, L. In vitro electrophysiological drug testing using human embryonic stem cell derived cardiomyocytes. Stem Cells Dev. 2009, 18, 161-172.

18. Reppel, M.; Pillekamp, F.; Brockmeier, K.; Matzkies, M.; Bekcioglu, A.; Lipke, T.; Nguemo, F.; Bonnemeier, H.; Hescheler, J. The electrocardiogram of human embryonic stem cell-derived cardiomyocytes. J. Electrocardiol. 2005, 38, 166-170.

19. Meyer, T.; Boven, K.H.; Gunther, E.; Fejtl, M. Micro-electrode arrays in cardiac safety pharmacology: A novel tool to study QT interval prolongation. Drug Saf. 2004, 27, 763-72.

20. Ayanda Biosystems SA. MEA Biochip Product Catalog. Available online: http://www.ayandabiosys.com/Documents/MEA_Product_Catalog.pdf (accessed on 3 September 2010).

21. MacCarthy, N.; Burke, M.; Alderman, J. A Low Cost High Resolution Fabrication Process for Disposable MEAs. In Proceedings of the 6th International Meeting on Substrate-Integrated Microelectrode Arrays, Reutlingen, Germany, 8-11 July 2008; pp. 321-323.

22. Ryynänen, T.; Kattipparambil Rajan, D.; Lekkala, J. Concept for Low-Cost Rapid Prototyping of New MEA Designs. In Proceedings of the 2009 Symposium on Microelectrode Arrays in Tissue Engineering (MEATE), Tampere, Finland, 4-5 June 2009.

23. Novak, J.L.; Wheeler, B.C. Recording from the Aplysia abdominal ganglion with a planar microelectrode array. IEEE Trans. Biomed. Eng. 1986, BME-33(February), 196-202.

24. Kim, J.S.; Lee, K.J.; Pak, J.J. The Effects of Pt Electroplating on the Multi-Electrode Array Surface in Measuring Neuronal Signal. Presented at APCTP-KU Joint Conference on Bio-complexity, Seoul, Republic of Korea, 2-5 November 2005; p. 10. Available online: http://mins.korea.ac.kr/bbs/?mid=conference\&page=5\&document_srl=1309 (accessed on 27 May 2011).

25. Gawad, S.; Giugliano, M.; Heuschkel, M.; Wessling, B.; Markram, H.; Schnakenberg, U.; Renaud, P.; Morgan, H. Substrate arrays of iridium oxide microelectrodes for in vitro neuronal intgerfacing. Front. Neuroeng. 2009, $2,1$.

26. Eick, S.; Wallys, J.; Hofmann, B.; van Ooyen, A.; Schnakenberg, U.; Ingebrandt, S.; Offenhäusser, A. Iridium oxide microelectrode arrays for in vitro stimulation of individual rat neurons from dissociated cultures. Front. Neuroeng. 2009, 2, 16.

27. Multi Channel Systems MCS GmbH. MEA Microelectrode (MEA) Manual. Available online: http://www.multichannelsystems.com/uploads/media/MEA_Manual.pdf (accessed on 23 May 2011).

28. Janders, M.; Egert, U.; Stelzle, M.; Nisch, W. Novel Thin Film Titanium Nitride Micro-Electrodes with Excellent Charge Transfer Capability for Cell Stimulation and Sensing Applications. In Proceeding of the 18th Annual International Conference of the IEEE Engineering in Medicine and Biology Society, Amsterdam, The Netherlands, 31 October-3 November 1996; pp. 245-247.

29. Egert, U.; Schlosshauer, B.; Fennrich, S.; Nisch, W.; Fejtl, M.; Knott, T.; Müller, T.; Hämmerle, H. A novel organotypic long-term culture of the rat hippocampus on substrate-integrated multielectrode arrays. Brain Res. Protoc. 1998, 2, 229-242.

30. Blau, A.; Ziegler, C.; Heyer, M.; Endres, F.; Schwitzgebel, G.; Matthies, T.; Stieglitz, T.; Meyer, J.U.; Gopel, W. Characterization and optimization of microelectrode arrays for in vivo nerve signal recording and stimulation. Biosens. Bioelectron. 1997, 12, 883-892. 
31. Weiland, J.D.; Humayun, M.S.; Anderson, D.J. In vitro electrical properties for iridium oxide versus titanium nitride stimulating electrodes. IEEE Trans. Biomed. Eng. 2002, 49, 1574-1579.

32. Wang, K.; Fishman, H.A.; Dai, H.; Harris, J.S. Neural stimulation with a carbon nanotube microelectrode array. Nano Lett. 2006, 6, 2043-2048.

33. Keefer, E.W.; Botterman, B.R.; Romero, M.I.; Rossi, A.F.; Gross, G.W. Carbon nanotube coating improves neuronal recordings. Nat. Nanotechnol. 2008, 3, 434-439.

34. David-Pur, M.; Shein, M.; Hanein, Y. Carbon nanotube-based neurochips. Meth. Mol. Biol. 2010, 625, 171-177.

35. Fuchsberger, K.; Le Goff, A.; Gerwig, R.; Burkhardt, C.; Elit, J.; Li, Y.; Scheipers, A.; Stett, A.; Stelzle, M. Integration of Carbon Nanotubes in Microelectrode Arrays by Microcontact Printing and Electropolymerization for Neurostimulation and Biosensing Applications. In Proceedings of the 7th International Meeting on Substrate-Integrated Microelectrode Arrays, Reutlingen, Germany, 29 June-2 July 2010; pp. 267-268.

36. Smart, S.K.; Cassady, A.I.; Lu, G.Q.; Martin, D.J. The biocompatibility of carbon nanotubes. Carbon 2006, 44, 1034-1047.

37. Boxley, C.J.; White, H.S.; Gardner, C.E.; Macpherson, J.V. Nanoscale imaging of the electronic conductivity of the native oxide film on titanium using conducting atomic force microscopy. J. Phys. Chem. B 2003, 107, 9677-9680.

38. Cai, K.; Müller, M.; Bossert, J.; Rechtenbach, A.; Jandt, K.D. Surface structure and composition of flat titanium thin films as a function of film thickness and evaporation rate. Appl. Surf. Sci. 2003, 250, 252-267.

39. McCafferty, E.; Wightman, J.P. An X-ray photoelectron spectroscopy sputter profile study of the native air-formed oxide film on titanium. Appl. Surf. Sci.1999, 143, 92-100.

40. Williams, K.R.; Gupta, K.; Wasilik, M. Etch rates for micromachining processing-Part II. J. Microelectromech. Syst. 2003, 12, 761-778.

41. Huigen, E.; Peper, A.; Grimbergen, C.A. Investigation into the origin of the noise of surface electrodes. Med. Biol. Eng. Comput. 2002, 40, 332-338.

42. Welch, P.D. The use of fast Fourier transform for the estimation of power spectra: A method based on time averaging over short, modified periodograms. IEEE Trans. Audio Electrocoust. 1967, $A U-15,70-78$.

43. Ihalainen, H. Matlab MSP Toolbox; Tampere University of Technology: Tampere, Finland, 1987-2010.

44. Agilent Technologies. Agilent Impedance Measurement Handbook: A Guide to Measurement Technology and Techniques; Agilent Technologies, Inc: Santa Clara, CA, USA, 2009. Available online: http://cp.literature.agilent.com/litweb/pdf/5950-3000.pdf (accessed on 11 May 2010).

45. Lappalainen, R.S.; Salomäki, M.; Ylä-Outinen, L.; Heikkilä, T.J.; Hyttinen, J.A.K.; Pihlajamäki, H.; Suuronen, R.; Skottman, H.; Narkilahti, S. Similarly derived and cultured hESC lines show variation in their developmental potential towards neuronal cells in long-time culture. Regen. Med. 2010, 5, 749-762. 
46. Sundberg, M.; Jansson, L.; Ketolainen, J.; Pihlajamäki, H.; Suuronen, R.; Skottman, H.; Inzunza, J.; Hovatta, O.; Narkilahti, S. CD marker expression profiles of human embryonic stem cells and their neural derivatives, determined using flow-cytometric analysis, reveal a novel CD marker for exclusion of pluripotent stem cells. Stem Cell Res. 2009, 2, 113-124.

47. Mummery, C.; Ward-van Oostwaard, D.; Doevendans, P.; Spijker, R.; van den Brink, S.; Hassink, R.; van der Heyden, M.; Opthof, T.; Pera, M.; de la Riviere, A.B.; et al. Differentiation of human embryonic stem cells to cardiomyocytes: Role of coculture with visceral endoderm-like cells. Circulation 2003, 107, 2733-2740.

48. Ryynänen, T.; Kujala, V.; Ylä-Outinen, L.; Kerkelä, E.; Narkilahti, S.; Lekkala, J. Polystyrene Coated MEA. In Proceedings of the 7th International Meeting on Substrate-Integrated Microelectrode Arrays, Reutlingen, Germany, 29 June-2 July 2010; pp. 265-266.

49. Blau, A.; Murr, A.; Trellenkamp, S.; Dautermann, C.; Wolff, S.; Heuschkel, M.; Wuesten, J.; Ziegler, C.; Benfenati, F. Prototyping All-Polymer Bioelectrical Signal Transducers. In Proceedings of the World Congress on Medical Physics and Biomedical Engineering, Munich, Germany, 7-12 September 2009; pp. 327-330.

(C) 2011 by the authors; licensee MDPI, Basel, Switzerland. This article is an open access article distributed under the terms and conditions of the Creative Commons Attribution license (http://creativecommons.org/licenses/by/3.0/). 